\title{
Editorial
}

\section{Semiconductors: Materials, Physics, and Devices}

\author{
Jiangwei Liu, ${ }^{1}$ Hongyang Zhao, ${ }^{2}$ Jinlong Liu, ${ }^{3}$ Aurélien Maréchal, ${ }^{1}$ and Wei Wang ${ }^{4}$ \\ ${ }^{1}$ Research Center for Functional Materials, National Institute for Materials Science, 1-1 Namiki, Tsukuba, Ibaraki 3050044, Japan \\ ${ }^{2}$ Department of Materials Science and Engineering, Wuhan Institute of Technology, Wuhan 430073, China \\ ${ }^{3}$ School of Materials Science and Engineering, University of Science and Technology Beijing, Beijing 100083, China \\ ${ }^{4}$ School of Electronic and Information Engineering, Xian Jiaotong University, Xian, Shaanxi 710049, China
}

Correspondence should be addressed to Jiangwei Liu; liu.jiangwei@nims.go.jp

Received 8 November 2016; Accepted 8 November 2016

Copyright (C) 2016 Jiangwei Liu et al. This is an open access article distributed under the Creative Commons Attribution License, which permits unrestricted use, distribution, and reproduction in any medium, provided the original work is properly cited.

The Si, GaAs, and InP based semiconductor devices have improved our lives significantly during the last century. Wide-bandgap semiconductors, such as $\mathrm{ZnO}, \mathrm{SiC}, \mathrm{GaN}$, $\mathrm{Ga}_{2} \mathrm{O}_{3}$, and diamond, are believed to be the next generation semiconductors because of their excellent physical and chemical properties. These semiconductors are promising candidates for fabrication of optoelectronic devices and semiconductor electronics, such as light emitters, solar cells, solar blind detectors, high-power electronics, gas sensors, highpower microwave transistors, and microelectromechanical system (MEMS). In order to fabricate high-performance semiconductor electronic and optoelectronic devices, several research fields are being developed, such as high quality bulk or epitaxial layer growth, $n$-type and $p$-type doping, surface crystal quality improvement, interface quality of metal/semiconductor or insulator/semiconductor junctions, optimization of device fabrication processes, and new application development for the semiconductor devices. During recent few years, significant progresses in semiconductor growth and device technology have been developed. It would be valuable and meaningful to realize the research situations of them.

In this special issue, A. Maerz et al. gave a general description of the thermal behavior of vertical $\mathrm{SiC}$ metaloxide-semiconductor field-effect transistor (MOSFET) under various driving and boundary conditions in case of a shortcircuit event. The accurate prediction of the SiC MOSFET withstanding time for single fault events greatly influences the requirements for device protection circuits for these devices in power converter applications, like voltage-sourceinverters or power-electronic-transformers. For this reason a thermal model based on the structural design, the physical dimensions of the chip, and material properties of $4 \mathrm{H}$ $\mathrm{SiC}$ is proposed. R. Banchuin analyzed random variation in subthreshold floating-gate MOSFET (FGMOSFET). The analysis of random variation in the performance of FGMOSFET which is an often cited semiconductor based electronic device, operated in the subthreshold region defined in terms of its drain current, has been proposed. C.-I. Lee et al. investigated noise parameters of silicon germanium ( $\mathrm{SiGe}$ ) heterojunction bipolar transistors (HBTs) for different sizes in the breakdown region for the first time. Good agreement between experimental and simulated noise performance at breakdown is achieved for different sized SiGe HBTs. The presented analysis can benefit the RF circuits operating in the breakdown region. $\mathrm{H}$. Lim et al. gave a review about the modeling of magnetic tunnel junction (MTJ). Modeling methods and models of MTJ characteristics are classified into two groups, macromodels and behavioral models, and the most important characteristics of MTJs. A. Hariya et al. demonstrated considerations of physical design and implementation for wide regulation range $\mathrm{MHz}$-level LLC resonant DC-DC converters with gallium nitride high electron mobility transistors. The physical parameters and size of the planar transformer are optimized by using derived equations and finite element method with Maxwell 3D.

We hope this special issue could provide the readers with an overview of the recent achievements in the field of semiconductor based materials, physics, and devices. We also hope that this special issue could provide a valuable reference for semiconductor researchers. 


\section{Acknowledgments}

We would like to thank all the contributing authors for the submission to this special issue. We also greatly appreciate the reviewers for their help in the review process for the special issue.

Jiangwei Liu Hongyang Zhao Jinlong Liu

Aurélien Maréchal Wei Wang 


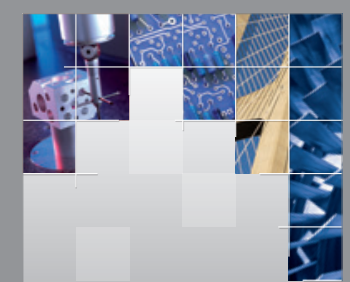

\section{Enfincering}
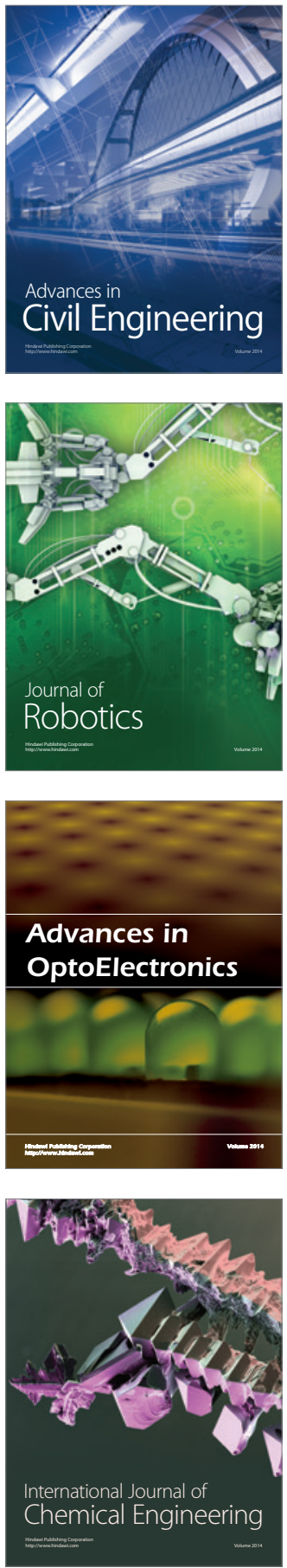

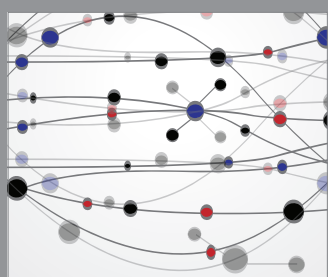

The Scientific World Journal

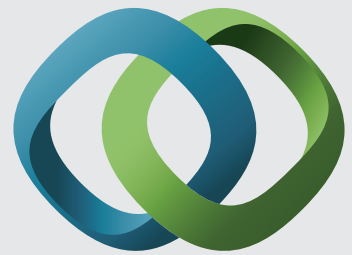

\section{Hindawi}

Submit your manuscripts at

http://www.hindawi.com
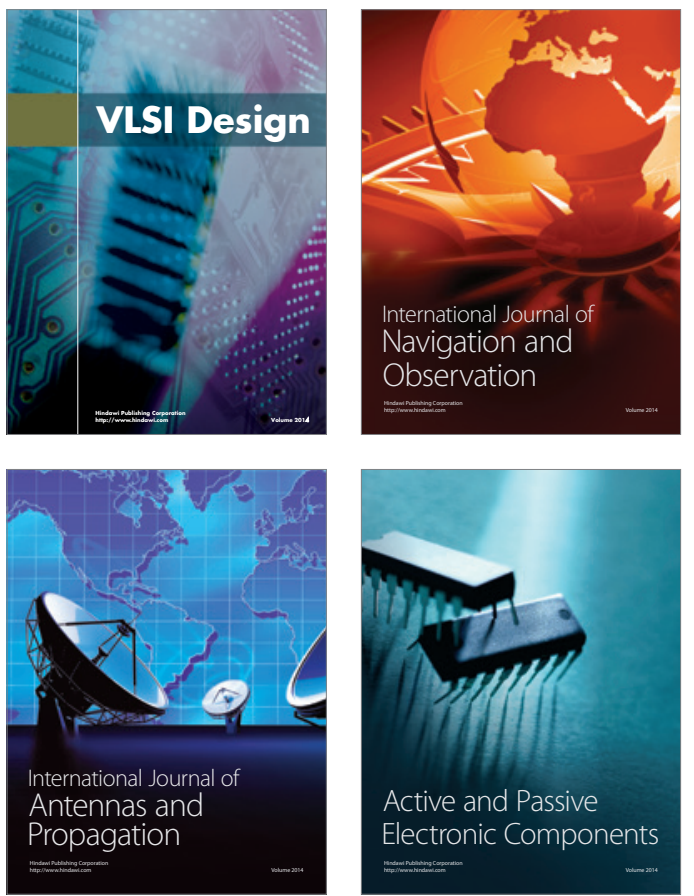
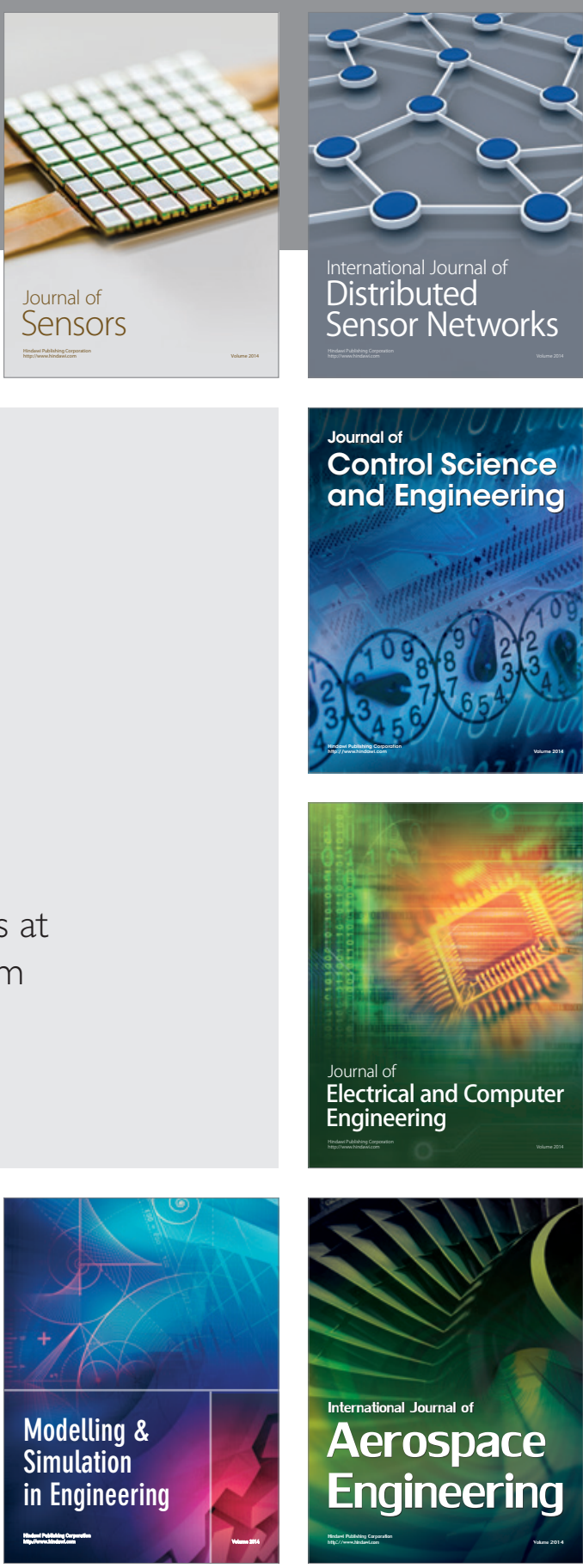

International Journal of

Distributed

Sensor Networks

Journal of

Control Science

and Engineering
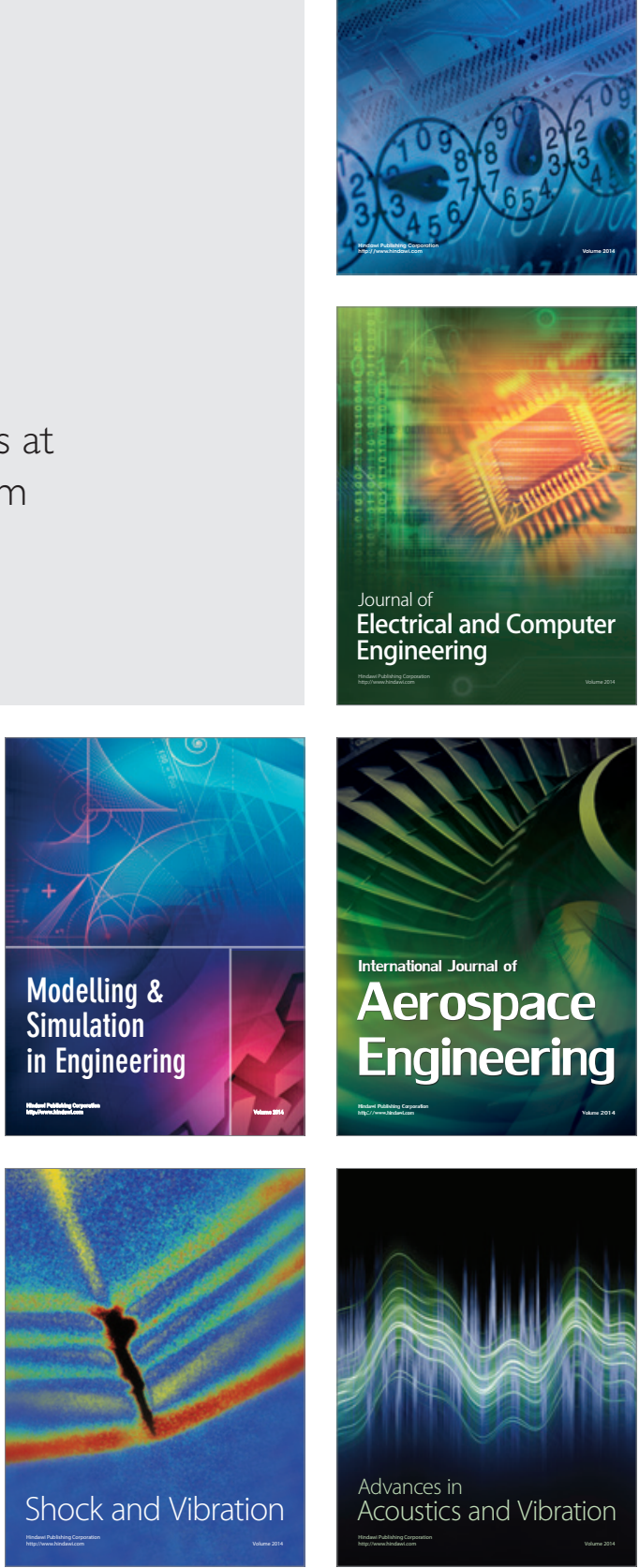